\title{
Characterizing inequality benchmark incomes
}

\author{
Laurence S. J. Roope $^{1}{ }_{(D)}$
}

Received: 8 February 2018 / Accepted: 28 May 2018 / Published online: 8 June 2018

(C) The Author(s) 2018

\begin{abstract}
Many inequality measures have the property that for any income distribution there exists a benchmark income, above which adding incremental income increases inequality, and below which it decreases inequality. This note provides social preference conditions which guarantee the existence of such a benchmark income. The key condition is a strong version of the Pigou-Dalton transfer principle. The results imply that benchmark incomes exist for virtually all known inequality measures.
\end{abstract}

Keywords Inequality · Inequality measurement · Benchmark income · Transfer principle

JEL Classification D31 · D63

\section{Introduction}

A number of studies have considered how incremental increases in income, at specific points in the income distribution, impact inequality. Lambert and Lanza (2006) established that, for all Lorenz-consistent inequality indices, a benchmark income or position exists, above which adding increments of income increases inequality, and below which it decreases inequality. They went on to derive, for a variety of 'relative' inequality indices, explicit results on where these critical points in an income distri-

$凶 \quad$ Laurence S. J. Roope

Laurence.Roope@dph.ox.ac.uk

1 Health Economics Research Centre, Nuffield Department of Population Health, University of Oxford, Oxford OX3 7LF, UK 
bution lie. ${ }^{1}$ Benchmark incomes/positions have also been derived for some 'absolute' measures (Corvalan 2014). ${ }^{2}$ These benchmark incomes can be interpreted as social reference levels for inequality, somewhat analogous to poverty lines, above which increases to income increase inequality, and below which they decrease inequality. Knowledge of the location of such a point in the income distribution could be used, for example, to predict the impact on inequality of a subsidy to income at a particular point in the distribution.

This note provides a theoretical foundation which embeds the concept of a benchmark income/position within a social preference framework. We assume a social preference ordering over all possible income distributions and impose intuitive ethical principles on the preference ordering. This gives rise to the existence of a benchmark income/position below which adding incremental income is socially desirable, and above which it is undesirable. The assumptions which ensure the existence of benchmark incomes/positions are sufficiently mild to guarantee that they must exist for virtually all inequality measures that have been proposed in the literature. The exact value of a critical point in a given income distribution will depend on the additional social preferences embodied by a given inequality measure, beyond those imposed in our axiomatic framework.

The rest of the paper is organized as follows. Some notation and the basic framework are provided in Sect. 2. Examples of specific benchmark incomes/positions implied by several well-known inequality measures are provided in Sect. 3. The paper's central results are derived in Sect. 4. Some concluding remarks are offered in Sect. 5. All proofs are deferred to the Appendices.

\section{Notation and basic framework}

Consider a society of $n \geq 2$ individuals. A profile $\mathbf{x}=\left(x_{1}, \ldots, x_{n}\right) \in \mathbb{R}_{+}^{n}$ represents the distribution of incomes within the society.

A social preference relation, denoted by the symbol $\succcurlyeq$, on $\mathbb{R}_{+}^{n}$ is assumed. As usual, $\succcurlyeq$ means weak preference, $\succ$ denotes strict preference, and $\sim$ is indifference. Sometimes we will use reversed preference symbols, $\preccurlyeq$ and $\prec$. We will assume that the preference relation is complete, i.e., for all $\mathbf{x}, \mathbf{y} \in \mathbb{R}_{+}^{n}, \mathbf{x} \preccurlyeq \mathbf{y}$ or $\mathbf{y} \preccurlyeq \mathbf{x} .^{3}$ We will also assume that the preference relation is transitive, i.e., for all $\mathbf{x}, \mathbf{y}, \mathbf{z} \in \mathbb{R}_{+}^{n}$, if $\mathbf{x} \succ \mathbf{y}$ and $\mathbf{y} \succ \mathbf{z}$ then $\mathbf{x} \succ \mathbf{z}$.

An inequality measure is a function that assigns to each income profile a nonnegative number. Thus $I: \bigcup_{n \in \mathbb{N}} \mathbb{R}_{+}^{n} \rightarrow \mathbb{R}_{+}$.

\footnotetext{
1 Lambert and Lanza (2006) describe this critical point as a "benchmark" income level or position in the distribution. Hoffmann (2001), who derives some of the same results, describes this critical point as a "relative poverty line."

2 'Relative' inequality measures are those which are invariant under equiproportional increases in all incomes. By contrast, 'absolute' inequality measures are those which register no change when the same absolute amount of income is added to all incomes. 'Centrist' inequality measures (sometimes also referred to as 'intermediate' or 'compromise' measures) register an increase in inequality if all incomes increase equiproportionally, and a decrease if the same absolute amount of income is added to all incomes.

${ }^{3}$ In our interpretation, completeness includes reflexivity, that is, for all $\mathbf{x} \in \mathbb{R}_{+}^{n}, \mathbf{x} \sim \mathbf{x}$.
} 
For a permutation $\rho$ of the indices in $\{1, \ldots, n\}$, such that $\rho_{i}:=\rho(i)$, we write $\rho(\mathbf{x})$ for the profile $\mathbf{z}$ with $z_{i}=x_{\rho_{i}}$ for $i=1, \ldots, n$. The preference relation $\succcurlyeq$ satisfies a property of symmetry (or anonymity): $\mathbf{x} \sim \rho(\mathbf{x})$ for all $\mathbf{x} \in \mathbb{R}_{+}^{n}$. From here on, for simplicity, when we write $\mathbf{x} \in \mathbb{R}_{+}^{n}$ it is implicitly assumed that $x_{1} \leq \cdots \leq x_{n}$. Since the relation $\succcurlyeq$ satisfies symmetry, this is not a restriction. Furthermore, we write $w_{i} \mathbf{x}$ for the profile $\mathbf{x}$ with $x_{i}$ replaced by $w$; whenever we use this notation we implicitly assume that the ordering of incomes remains from lowest to highest, i.e., $x_{i-1} \leq w \leq x_{i+1}$ if $i \in\{2, \ldots, n-1\}, x_{i-1} \leq w$ if $i=n$, and $w \leq x_{i+1}$ if $i=1$. For example, for $\varepsilon \geq 0$ when we write $\left(x_{i}+\varepsilon\right)_{i} \mathbf{x}$, we implicitly require constraints on the admissible values of $\varepsilon$, so that $x_{i}+\varepsilon \leq x_{i+1}$ whenever $i \in\{1, \ldots, n-1\}$; in particular, $\varepsilon=0$ if $x_{i}=x_{i+1}$. Whenever we write $\left(x_{i}+\varepsilon\right)_{i} \mathbf{x}$ with $\varepsilon>0$ it is implicitly assumed that $x_{i}<x_{i+1}$ if $i \neq n$.

Finally, we denote the mean of profile $\mathbf{x} \in \mathbb{R}_{+}^{n}$ by $\mu=\frac{1}{n} \cdot \sum_{i=1}^{n} x_{i}$.

\section{Examples of inequality benchmark incomes}

To further motivate our interest in characterizng the existence of an inequality benchmark income, this section provides some specific examples of inequality benchmark incomes. These examples will demonstrate that benchmark incomes exist for a wide range of, but not all, inequality measures.

In Proposition 1 we will consider five inequality measures, defined as follows. The Gini coefficient is given by:

$$
I_{G}(\mathbf{x})=1-\frac{1}{n}\left[\frac{\sum_{k=1}^{n} 2\left(n-k+\frac{1}{2}\right) x_{k}}{\sum_{i=1}^{n} x_{i}}\right] .
$$

The mean log deviation is given by:

$$
I_{T}(\mathbf{x})=\frac{1}{n} \sum_{i=1}^{n} \ln \left(\frac{\mu}{x_{i}}\right)
$$

The absolute Gini is given by:

$$
I_{A G}(\mathbf{x})=\mu \cdot I_{G}(\mathbf{x})
$$

The variance is given by: ${ }^{4}$

$$
I_{V}(\mathbf{x})=\frac{1}{n} \sum_{i=1}^{n}\left(x_{i}-\mu\right)^{2}
$$

\footnotetext{
${ }^{4}$ Occasionally, where it is not explicitly being interpreted as an inequality indicator, the variance will be denoted simply by $\sigma_{\mathbf{x}}^{2}$.
} 
The Krtscha is given by:

$$
I_{K}(\mathbf{x})=\frac{1}{n \mu} \sum_{i=1}^{n}\left(x_{i}-\mu\right)^{2} .
$$

These five measures include two 'relative' measures, $I_{G}(\cdot)$ and $I_{T}(\cdot)$; two 'absolute' measures $I_{A G}(\cdot)$ and $I_{V}(\cdot)$; and a 'centrist' measure $I_{K}(\cdot)$. For any inequality measure $I(\cdot)$, let $\triangle I(\mathbf{x} ; l, \varepsilon)=I\left(\left(x_{l}+\varepsilon\right)_{l} \mathbf{x}\right)-I(\mathbf{x})$. This can be interpreted as the change in inequality associated with an incremental increase in individual $l$ 's income. We can now state the following result. ${ }^{5}$

Proposition 1 Given any profile $\mathbf{x} \in \mathbb{R}_{+}^{n}$, any $l \in\{1, \ldots, n\}$, and any $\varepsilon>0$, the following hold:

(i) $\triangle I_{G}(\mathbf{x} ; l, \varepsilon)>0$ if and only if $l>\frac{\sum_{k=1}^{n} k x_{k}}{\sum_{i=1}^{n} x_{i}} ; c_{\mathbf{x}}=\frac{\sum_{k=1}^{n} k x_{k}}{\sum_{i=1}^{n} x_{i}}$ is a benchmark income.

(ii) $\triangle I_{T}(\mathbf{x} ; l, \varepsilon)>0$ if and only if $x_{l}>\frac{\varepsilon}{\left(1+\frac{\varepsilon}{n \mu}\right)^{n}-1} ; c_{\mathbf{x}, \varepsilon}=\frac{\varepsilon}{\left(1+\frac{\varepsilon}{n \mu}\right)^{n}-1}$ is a benchmark income and $\lim _{\varepsilon \rightarrow 0} c_{\mathbf{x}, \varepsilon}=\mu$.

(iii) $\triangle I_{A G}(\mathbf{x} ; l, \varepsilon)>0$ if and only if $l>\frac{n+1}{2} ; c_{\mathbf{x}}=\frac{n+1}{2}$ is a benchmark income.

(iv) $\triangle I_{V}(\mathbf{x} ; l, \varepsilon)>0$ if and only if $x_{l}>\mu+\frac{1}{2}\left(\frac{n-1}{n}\right) \varepsilon ; c_{\mathbf{x}, \varepsilon}=\mu+\frac{1}{2}\left(\frac{n-1}{n}\right) \varepsilon$ is a benchmark income and $\lim _{\varepsilon \rightarrow 0} c_{\mathbf{x}, \varepsilon}=\mu$.

(v) $\triangle I_{K}(\mathbf{x} ; l, \varepsilon)>0$ if and only if $x_{l}>\mu+\frac{\sigma_{\mathbf{x}}^{2}}{2 \mu}-\frac{\varepsilon(n-1)}{2 n} ; c_{\mathbf{x}, \varepsilon}=\mu+\frac{\sigma_{\mathbf{x}}^{2}}{2 \mu}-\frac{\varepsilon(n-1)}{2 n}$ is a benchmark income and $\lim _{\varepsilon \rightarrow 0} c_{\mathbf{x}, \varepsilon}=\mu+\frac{\sigma_{\mathbf{x}}^{2}}{2 \mu}$.

\section{Proof See Appendix A.}

Proposition 1 illustrates that benchmark incomes exist for inequality measures with widely varying normative underpinnings. However, benchmark incomes do not exist for all inequality measures that have been proposed in the literature. Consider, for example, the interquartile range given by:

$$
I_{I Q R}(\mathbf{x})=q_{3}-q_{1},
$$

where $q_{i}$ denotes quartile $i$. It is easy to show via a counterexample that a benchmark income does not exist for $I_{I Q R}(\cdot)$. Consider the profile $\mathbf{x}=(1,3,5,6,7,9,12)$. Here $I_{I Q R}(\mathbf{x})=9-3=6$, being determined, respectively, by incomes $x_{2}=3\left(q_{1}\right)$ and $x_{6}=9\left(q_{3}\right)$. It is clear that no benchmark income exists. For example, increasing $x_{2}$ from three to four would reduce inequality according to $I_{I Q R}(\cdot)$ from six to five, yet increasing $x_{1}$ from one to two would leave inequality unchanged at $I_{I Q R}(\cdot)=6$.

\footnotetext{
5 Some minor technical differences aside, parts (i) and (ii) of Proposition 1 have already been proven by Hoffmann (2001) and Lambert and Lanza (2006), and part (iii) has been proven by Corvalan (2014).
} 


\section{The existence of inequality benchmark incomes}

The following axioms impose constraints on the social preference relation $\succcurlyeq$. The first axiom is a strong version of the well-known Pigou-Dalton transfer principle. It simply says that, ceteris paribus, a progressive transfer of income, from a better-off individual to a less well-off individual, always results in a new income distribution which is socially preferable to the original one.

Axiom 1 (Strong Transfer): The preference $\succcurlyeq$ on $\mathbb{R}_{+}^{n}$ satisfies strong transfer if for all profiles $\mathbf{x} \in \mathbb{R}_{+}^{n}$ and all $\varepsilon>0,\left(x_{i}+\varepsilon\right)_{i}\left(x_{j}-\varepsilon\right)_{j} \mathbf{x} \succ \mathbf{x}$ for all $x_{i}<x_{j}$.

This axiom is widely considered a cornerstone of inequality measurement. Virtually all inequality measures that have been proposed in the literature are consistent with this social preference property. ${ }^{6}$

The second axiom imposes a consistency requirement on the social preference relation. Consider a situation where an individual's income, say $x_{i}$, increases by a certain amount. Suppose that this increase leads to no improvement in the social ranking of the resulting income profile compared to the original one. The axiom says that if this is the case, then no social progress would have been made by increasing this individual's income by some other amount. Conversely, suppose that some increase in an individual's income does lead to an improvement in the social ranking of the resulting income profile compared to the original one. Then increasing that individual's income by some different amount should also lead to some social improvement.

Axiom 2 (Positional Consistency): The preference $\succcurlyeq$ on $\mathbb{R}_{+}^{n}$ satisfies positional consistency if for all profiles $\mathbf{x} \in \mathbb{R}_{+}^{n}$ and all individuals $i \in\{1, \ldots, n\}$, we have:

(i) $\left(x_{i}+\varepsilon\right)_{i} \mathbf{x} \succ \mathbf{x}$ for some $\varepsilon>0 \Rightarrow\left(x_{i}+\delta\right)_{i} \mathbf{x} \succ \mathbf{x}$, for all $\delta>0$.

(ii) $\left(x_{i}+\varepsilon\right)_{i} \mathbf{x} \preccurlyeq \mathbf{x}$ for some $\varepsilon>0 \Rightarrow\left(x_{i}+\delta\right)_{i} \mathbf{x} \preccurlyeq \mathbf{x}$, for all $\delta>0$.

This axiom captures a social preference property embodied by an important subclass of inequality measures, namely rank-dependent measures which, as discussed, for example, by Lambert and Lanza (2006), depend not on income shares alone, but also on ranks. Such measures include the Gini and absolute Gini coefficients. By contrast, the property is not satisfied by rank-independent inequality measures, such as the mean log deviation, variance, or Krtscha.

We can now state and prove the paper's two main results, which provide conditions that ensure the existence of a benchmark income. It is clear from the results in Proposition 1 that, for a given inequality measure, the precise value of the benchmark income typically depends on the particular income profile $\mathbf{x} \in \mathbb{R}_{+}^{n}$. Consistent with this, in the following results, the proposed axioms guarantee the existence of a benchmark income which depends on the particular profile $\mathbf{x} \in \mathbb{R}_{+}^{n}$.

\footnotetext{
6 Exceptions include the rarely used interquartile range and relative mean deviation. As discussed by Atkinson (1983, p.23), any measures which, like these, are not strictly concave, cannot satisfy strong transfer. The relative mean deviation, for example, is unaffected by transfers between two incomes on the same side of the mean.
} 
Theorem 1 Assume that $\succcurlyeq$ is a complete, transitive, and symmetric preference relation on $\mathbb{R}_{+}^{n}$ which satisfies strong transfer. Then, for each $\mathbf{x} \in \mathbb{R}_{+}^{n}$ and $\delta>0$ there exists $c_{\mathbf{x}, \delta}$ such that (1) If $x_{i}<c_{\mathbf{x}, \delta}$ we have $\left(x_{i}+\delta\right)_{i} \mathbf{x} \succ \mathbf{x}$ and (2) If $x_{i}>c_{\mathbf{x}, \delta}$ we have $\left(x_{i}+\delta\right)_{i} \mathbf{x} \prec \mathbf{x}$.

\section{Proof See Appendix B.}

In Theorem 1, only increases in incomes below $c_{\mathbf{x}, \delta}$ are socially preferable. Note the dependence here of the critical income on the size of the income increment that is added. Such preferences are embodied, for example, by rank-independent inequality measures. Theorem 2 provides a similar result, applicable to preferences which, in addition to strong transfer, also satisfy the positional consistency requirement. In Theorem 2, only increases in incomes below $c_{\mathbf{x}}$ are socially preferable; this is true regardless of the size of the increase, as long as the rank ordering of incomes is maintained.

Theorem 2 Assume that $\succcurlyeq$ is a complete, transitive, and symmetric preference relation on $\mathbb{R}_{+}^{n}$ which satisfies strong transfer and positional consistency. Then, for each $\mathbf{x} \in \mathbb{R}_{+}^{n}$ there exists $c_{\mathbf{x}}$ such that (1) for all $x_{i}<c_{\mathbf{x}}$ we have $\left(x_{i}+\delta\right)_{i} \mathbf{x} \succ \mathbf{x}$ for all $\delta>0$ and (2) for all $x_{i}>c_{\mathbf{x}}$ we have $\left(x_{i}+\delta\right)_{i} \mathbf{x} \prec \mathbf{x}$ for all $\delta>0$.

\section{Proof See Appendix C.}

Remark 1 The benchmark income $c_{\mathbf{x}}$ in Theorem 2 is not unique. However, it can be inferred from the proof of Theorem 2 that, given any $\mathbf{x} \in \mathbb{R}_{+}^{n}$, any two benchmark incomes that are not equal must be sufficiently close to each other that each income $x_{i}$ is either greater than both benchmark incomes, or less than both benchmark incomes. No individual's income can lie betwen two different benchmark incomes.

\section{Discussion}

This note provides social preference axioms which guarantee the existence of a benchmark income/position below which adding incremental income is socially desirable, and above which it is undesirable. Together, Theorems 1 and 2 show that the key ingredient needed to guarantee the existence of such a benchmark income/position is simply a strong version of the ubiquitous Pigou-Dalton transfer property. Since this property underpins virtually all known inequality indices, so too the existence of benchmark incomes must be implied by virtually all inequality indices. The results can also be interpreted as indicating that the essence of a preference for the existence of a benchmark income/position with these properties is a strong concern for inequality. For example, such a benchmark income/position is inconsistent with weak monotonicity, which is a standard assumption in social welfare functions. It is also inconsistent with social preferences solely concerned about poverty. Increasing the incomes of those above the poverty line has traditionally been considered to leave the level of poverty in a society unchanged; following a seminal paper by Sen (1976), this intuition has been formalized in axiomatic work via the popular 'focus' axiom. In contrast, adding increments of income to those above this paper's benchmark incomes, 
leads to socially less preferred outcomes, capturing the intuition that there is someting socially undesirable about inequality.

Acknowledgements I am very grateful to Andrew Berg, Indranil Dutta, John McHale, and Jonathan Temple for their helpful comments and discussions. My thinking on the topic has also benefited from discussions on closely related issues with Kaushik Basu, Shasi Nandeibam, and Horst Zank-I am indebted to each of them. I am also grateful to an anonymous referee for making some excellent suggestions during the review process. Last but not least, the paper owes much to discussions with various participants at the CSAE Conference 2015: Economic Development in Africa, held at the University of Oxford during 22-24 March 2015, the 72nd European Meeting of the Econometric Society, held in Lisbon during 21-25 August 2017 and at seminars at ETH Zürich, the University of Oxford and the National University of Ireland, Galway. Naturally, any errors are the responsibility of myself alone.

Open Access This article is distributed under the terms of the Creative Commons Attribution 4.0 International License (http://creativecommons.org/licenses/by/4.0/), which permits unrestricted use, distribution, and reproduction in any medium, provided you give appropriate credit to the original author(s) and the source, provide a link to the Creative Commons license, and indicate if changes were made.

\section{Appendices}

\section{A Proof of Proposition 1}

Part (i). Take an arbitrary $\mathbf{x} \in \mathbb{R}_{+}^{n}, l \in\{1, \ldots, n\}$, and $\varepsilon>0$. It follows from (1) that

$$
I_{G}\left(\left(x_{l}+\varepsilon\right)_{l} \mathbf{x}\right)=1-\frac{1}{n}\left[\frac{\sum_{k=1, k \neq l}^{n} 2\left(n-k+\frac{1}{2}\right) x_{k}+2\left(n-l+\frac{1}{2}\right)\left(x_{l}+\varepsilon\right)}{\varepsilon+\sum_{i=1}^{n} x_{i}}\right] .
$$

By definition, $\Delta I_{G}(\mathbf{x} ; l, \varepsilon)=I_{G}\left(\left(x_{l}+\varepsilon\right)_{l} \mathbf{x}\right)-I_{G}(\mathbf{x})$.

It follows that:

$$
\begin{aligned}
\Delta I_{G}(\mathbf{x} ; l, \varepsilon) & \\
= & -\frac{1}{n}\left[\frac{\left.\sum_{i=1}^{n} x_{i}\left(\sum_{k=1, k \neq l}^{n} 2\left(n-k+\frac{1}{2}\right) x_{k}+2\left(n-l+\frac{1}{2}\right)\left(x_{l}+\varepsilon\right)\right)\right]}{\left(\varepsilon+\sum_{i=1}^{n} x_{i}\right) \sum_{i=1}^{n} x_{i}}\right] \\
& +\frac{1}{n}\left[\frac{\left.\left(\varepsilon+\sum_{i=1}^{n} x_{i}\right) \sum_{k=1}^{n} 2\left(n-k+\frac{1}{2}\right) x_{k}\right]}{\left(\varepsilon+\sum_{i=1}^{n} x_{i}\right) \sum_{i=1}^{n} x_{i}}\right] \\
\Delta I_{G}(\mathbf{x} ; l, \varepsilon) & \\
= & \frac{\left(\varepsilon+\sum_{i=1}^{n} x_{i}\right) \sum_{k=1}^{n} 2\left(n-k+\frac{1}{2}\right) x_{k}-\sum_{i=1}^{n} x_{i}\left(\sum_{k=1}^{n} 2\left(n-k+\frac{1}{2}\right) x_{k}+2\left(n-l+\frac{1}{2}\right) \varepsilon\right)}{n\left(\varepsilon+\sum_{i=1}^{n} x_{i}\right) \sum_{i=1}^{n} x_{i}} \\
= & \frac{\varepsilon \sum_{k=1}^{n} 2\left(n-k+\frac{1}{2}\right) x_{k}-2\left(n-l+\frac{1}{2}\right) \varepsilon \sum_{i=1}^{n} x_{i}}{n\left(\varepsilon+\sum_{i=1}^{n} x_{i}\right) \sum_{i=1}^{n} x_{i}} \\
= & \frac{\varepsilon\left[\sum_{k=1}^{n} 2\left(n-k+\frac{1}{2}\right) x_{k}-2\left(n-l+\frac{1}{2}\right) \sum_{i=1}^{n} x_{i}\right]}{n\left(\varepsilon+\sum_{i=1}^{n} x_{i}\right) \sum_{i=1}^{n} x_{i}} \\
= & \frac{\varepsilon\left[-2 \sum_{k=1}^{n} k x_{k}+2 l \sum_{i=1}^{n} x_{i}\right]}{n\left(\varepsilon+\sum_{i=1}^{n} x_{i}\right) \sum_{i=1}^{n} x_{i}}
\end{aligned}
$$




$$
=\frac{2 \varepsilon\left[l \sum_{i=1}^{n} x_{i}-\sum_{k=1}^{n} k x_{k}\right]}{n\left(\varepsilon+\sum_{i=1}^{n} x_{i}\right) \sum_{i=1}^{n} x_{i}} .
$$

The expression in (7) is (strictly) positive if and only if:

$$
l \sum_{i=1}^{n} x_{i}>\sum_{k=1}^{n} k x_{k}
$$

yielding the necessary and sufficient condition:

$$
l>\frac{x_{1}+2 x_{2}+\cdots+n x_{n}}{x_{1}+\cdots+x_{n}}=\frac{\sum_{k=1}^{n} k x_{k}}{\sum_{i=1}^{n} x_{i}} .
$$

Thus $c_{\mathbf{x}}=\frac{\sum_{k=1}^{n} k x_{k}}{\sum_{i=1}^{n} x_{i}}$ is a benchmark income. This concludes part (i).

Part (ii). Take an arbitrary $\mathbf{x} \in \mathbb{R}_{+}^{n}, l \in\{1, \ldots, n\}$, and $\varepsilon>0$. It follows from (2) that

$$
\begin{aligned}
\triangle I_{T}(\mathbf{x} ; l, \varepsilon)= & \frac{1}{n}\left[\sum_{i=1, i \neq l}^{n}\left(\ln \left(\mu+\frac{\varepsilon}{n}\right)-\ln \left(x_{i}\right)\right)-\sum_{i=1}^{n}\left(\ln (\mu)-\ln \left(x_{i}\right)\right)\right] \\
& +\frac{1}{n}\left[\ln \left(\mu+\frac{\varepsilon}{n}\right)-\ln \left(x_{l}+\varepsilon\right)\right] \\
= & \frac{1}{n} \sum_{i=1}^{n} \ln \left(\mu+\frac{\varepsilon}{n}\right)-\frac{1}{n} \sum_{i=1}^{n}\left(\ln (\mu)-\ln \left(x_{i}\right)\right) \\
& -\frac{1}{n} \sum_{i=1, i \neq l}^{n} \ln \left(x_{i}\right)-\frac{1}{n} \ln \left(x_{l}+\varepsilon\right) \\
= & \frac{1}{n}\left[\sum_{i=1}^{n}\left(\ln \left(\mu+\frac{\varepsilon}{n}\right)-\ln (\mu)+\ln \left(x_{i}\right)\right)\right] \\
& -\frac{1}{n} \sum_{i=1}^{n} \ln \left(x_{i}\right)+\frac{1}{n} \ln \left(x_{l}\right)-\frac{1}{n} \ln \left(x_{l}+\varepsilon\right) \\
= & \frac{1}{n}\left[\sum_{i=1}^{n}\left(\ln \left(\mu+\frac{\varepsilon}{n}\right)-\ln (\mu)\right)\right]+\frac{1}{n}\left(\ln \left(x_{l}\right)-\ln \left(x_{l}+\varepsilon\right)\right) \\
= & \ln \left(\mu+\frac{\varepsilon}{n}\right)-\ln (\mu)+\frac{1}{n}\left(\ln \left(x_{l}\right)-\ln \left(x_{l}+\varepsilon\right)\right) .
\end{aligned}
$$

We then have:

$$
\begin{aligned}
\Delta I_{T}(\mathbf{x} ; l, \varepsilon)>0 & \Longleftrightarrow \ln \left(\mu+\frac{\varepsilon}{n}\right)-\ln (\mu)>\frac{1}{n}\left[\ln \left(x_{l}+\varepsilon\right)-\ln \left(x_{l}\right)\right] \\
& \Longleftrightarrow \ln \left(x_{l}+\varepsilon\right)-\ln \left(x_{l}\right)<n\left[\ln \left(\mu+\frac{\varepsilon}{n}\right)-\ln (\mu)\right]
\end{aligned}
$$




$$
\begin{aligned}
& \Longleftrightarrow \ln \left(\frac{x_{l}+\varepsilon}{x_{l}}\right)<n \ln \left(\frac{\mu+\frac{\varepsilon}{n}}{\mu}\right) \\
& \Longleftrightarrow \ln \left(\frac{x_{l}+\varepsilon}{x_{l}}\right)<n \ln \left(1+\frac{\varepsilon}{n \mu}\right) \\
& \Longleftrightarrow \ln \left(\frac{x_{l}+\varepsilon}{x_{l}}\right)<\ln \left(1+\frac{\varepsilon}{n \mu}\right)^{n} \\
& \Longleftrightarrow \frac{x_{l}+\varepsilon}{x_{l}}<\left(1+\frac{\varepsilon}{n \mu}\right)^{n} \\
& \Longleftrightarrow 1+\frac{\varepsilon}{x_{l}}<\left(1+\frac{\varepsilon}{n \mu}\right)^{n} \\
& \Longleftrightarrow \frac{\varepsilon}{x_{l}}<\left(1+\frac{\varepsilon}{n \mu}\right)^{n}-1 \\
& \Longleftrightarrow \frac{x_{l}}{\varepsilon}>\frac{1}{\left(1+\frac{\varepsilon}{n \mu}\right)^{n}-1} \\
& \Longleftrightarrow x_{l}>\frac{\varepsilon}{\left(1+\frac{\varepsilon}{n \mu}\right)^{n}-1} .
\end{aligned}
$$

Thus $c_{\mathbf{x}, \varepsilon}=\frac{\varepsilon}{\left(1+\frac{\varepsilon}{n \mu}\right)^{n}-1}$ is a benchmark income. It follows immediately that $\lim _{\varepsilon \rightarrow 0} c_{\mathbf{x}, \varepsilon}=\mu$, since function $c_{\mathbf{x}, \varepsilon}(\varepsilon)=\frac{\varepsilon}{\left(1+\frac{\varepsilon}{n \mu}\right)^{n}-1}$ is of the form $f(\varepsilon)=$ $\frac{\varepsilon}{\left(1+\frac{\varepsilon}{y}\right)^{z}-1}$, where $y=n \mu$ and $z=n$, and it is a standard result that $\lim _{\varepsilon \rightarrow 0} f(\varepsilon)=$ $\frac{y}{z}=\mu$.

This concludes the proof of part (ii).

Part (iii). Take an arbitrary $\mathbf{x} \in \mathbb{R}_{+}^{n}, l \in\{1, \ldots, n\}$, and $\varepsilon>0$. It follows from (3) that

$$
\begin{aligned}
\triangle & I_{A G}(\mathbf{x} ; l, \varepsilon)=\mu\left[I_{G}\left(x_{l}+\varepsilon\right)_{l} \mathbf{x}-I_{G}(\mathbf{x})\right]+\frac{\varepsilon}{n} I_{G}\left(x_{l}+\varepsilon\right)_{l} \mathbf{x} \\
= & \frac{\mu \varepsilon\left[\sum_{k=1}^{n} 2\left(n-k+\frac{1}{2}\right) x_{k}-2\left(n-l+\frac{1}{2}\right) \sum_{i=1}^{n} x_{i}\right]}{n\left(\varepsilon+\sum_{i=1}^{n} x_{i}\right) \sum_{i=1}^{n} x_{i}} \\
& +\frac{\varepsilon}{n}-\frac{\varepsilon}{n^{2}}\left[\frac{\left.\sum_{k=1, k \neq l}^{n} 2\left(n-k+\frac{1}{2}\right) x_{k}+2\left(n-l+\frac{1}{2}\right)\left(x_{l}+\varepsilon\right)\right]}{\varepsilon+\sum_{i=1}^{n} x_{i}}\right] \\
\triangle & I_{A G}(\mathbf{x} ; l, \varepsilon)=\frac{\mu \varepsilon\left[\sum_{k=1}^{n} 2\left(n-k+\frac{1}{2}\right) x_{k}-2\left(n-l+\frac{1}{2}\right) \sum_{i=1}^{n} x_{i}\right]}{n\left(\varepsilon+\sum_{i=1}^{n} x_{i}\right) \sum_{i=1}^{n} x_{i}}+\frac{\varepsilon}{n} \\
& -\frac{\varepsilon}{n^{2}}\left[\frac{\sum_{k=1}^{n} 2\left(n-k+\frac{1}{2}\right) x_{k}+2\left(n-l+\frac{1}{2}\right) \varepsilon}{\varepsilon+\sum_{i=1}^{n} x_{i}}\right] \\
= & \frac{\mu \varepsilon\left[\sum_{k=1}^{n} 2\left(n-k+\frac{1}{2}\right) x_{k}-2\left(n-l+\frac{1}{2}\right) n \mu\right]}{n(\varepsilon+n \mu) n \mu}
\end{aligned}
$$




$$
\begin{aligned}
& +\frac{\varepsilon}{n}-\frac{\varepsilon}{n^{2}}\left[\frac{\sum_{k=1}^{n} 2\left(n-k+\frac{1}{2}\right) x_{k}+2\left(n-l+\frac{1}{2}\right) \varepsilon}{\varepsilon+n \mu}\right] \\
& =\frac{\mu \varepsilon\left[2 n \sum_{k=1}^{n} x_{k}-2 \sum_{k=1}^{n} k x_{k}+n \mu-2 n^{2} \mu+2 \ln \mu-n \mu\right]}{n^{2} \mu(\varepsilon+n \mu)}+\frac{\varepsilon}{n} \\
& -\frac{\varepsilon}{n^{2}}\left[\frac{2 n \sum_{k=1}^{n} x_{k}-2 \sum_{k=1}^{n} k x_{k}+n \mu+2 n \varepsilon-2 l \varepsilon+\varepsilon}{\varepsilon+n \mu}\right] \\
& =\frac{\mu \varepsilon\left[2 n^{2} \mu-2 \sum_{k=1}^{n} k x_{k}-2 n^{2} \mu+2 \ln \mu\right]}{n^{2} \mu(\varepsilon+n \mu)}+\frac{\varepsilon}{n} \\
& -\frac{\varepsilon}{n^{2}}\left[\frac{2 n^{2} \mu-2 \sum_{k=1}^{n} k x_{k}+n \mu+2 n \varepsilon-2 l \varepsilon+\varepsilon}{\varepsilon+n \mu}\right] \\
& =\frac{\varepsilon}{n}+\frac{-2 \varepsilon \sum_{k=1}^{n} k x_{k}+2 \varepsilon \ln \mu-2 \varepsilon \mu n^{2}+2 \varepsilon \sum_{k=1}^{n} k x_{k}-\varepsilon n \mu-2 n \varepsilon^{2}+2 l \varepsilon^{2}-\varepsilon^{2}}{n^{2}(\varepsilon+n \mu)} \\
& =\frac{\varepsilon}{n}+\frac{2 \varepsilon \ln \mu-2 \varepsilon \mu n^{2}-\varepsilon n \mu-2 n \varepsilon^{2}+2 l \varepsilon^{2}-\varepsilon^{2}}{n^{2}(\varepsilon+n \mu)} \\
& =\frac{\varepsilon n(\varepsilon+n \mu)+2 \varepsilon \ln \mu-2 \varepsilon \mu n^{2}-\varepsilon n \mu-2 n \varepsilon^{2}+2 l \varepsilon^{2}-\varepsilon^{2}}{n^{2}(\varepsilon+n \mu)} \\
& =\frac{\varepsilon^{2} n+\varepsilon n^{2} \mu+2 \varepsilon \ln \mu-2 \varepsilon \mu n^{2}-\varepsilon n \mu-2 n \varepsilon^{2}+2 l \varepsilon^{2}-\varepsilon^{2}}{n^{2}(\varepsilon+n \mu)} \\
& =\frac{-\varepsilon^{2} n+\varepsilon n^{2} \mu+2 \varepsilon \ln \mu-2 \varepsilon \mu n^{2}-\varepsilon n \mu+2 l \varepsilon^{2}-\varepsilon^{2}}{n^{2}(\varepsilon+n \mu)} \\
& =\frac{-\varepsilon^{2} n-\varepsilon \mu n^{2}+\varepsilon n \mu(2 l-1)+\varepsilon^{2}(2 l-1)}{n^{2}(\varepsilon+n \mu)} \\
& =\frac{(2 l-1)\left(\varepsilon n \mu+\varepsilon^{2}\right)-\left(\varepsilon^{2} n+\varepsilon \mu n^{2}\right)}{n^{2}(\varepsilon+n \mu)} \\
& =\frac{(2 l-1) \varepsilon(n \mu+\varepsilon)-\varepsilon n(\varepsilon+\mu n)}{n^{2}(\varepsilon+n \mu)} \\
& =\frac{\varepsilon(2 l-1-n)}{n^{2}} \text {. }
\end{aligned}
$$

We then have that

$$
\triangle I_{A G}(\mathbf{x} ; l, \varepsilon)>0 \Longleftrightarrow l>\frac{n+1}{2} .
$$

Thus $c_{\mathbf{X}}=\frac{n+1}{2}$ is a benchmark income. This concludes the proof of part (iii).

$\operatorname{Part}(i v)$. Take an arbitrary $\mathbf{x} \in \mathbb{R}_{+}^{n}, l \in\{1, \ldots, n\}$, and $\varepsilon>0$. It follows from (4) that

$$
\begin{aligned}
& I_{V}\left(\left(x_{l}+\varepsilon\right)_{l} \mathbf{x}\right) \\
& \quad=\frac{1}{n}\left[\sum_{i=1, i \neq l}^{n}\left(x_{i}-\left(\mu+\frac{\varepsilon}{n}\right)\right)^{2}+\left(x_{l}+\varepsilon-\left(\mu+\frac{\varepsilon}{n}\right)\right)^{2}\right]
\end{aligned}
$$




$$
\begin{aligned}
= & \frac{1}{n}\left[\sum_{i=1, i \neq l}^{n}\left(x_{i}^{2}-2 x_{i}\left(\mu+\frac{\varepsilon}{n}\right)+\mu^{2}+\frac{2 \mu \varepsilon}{n}+\frac{\varepsilon^{2}}{n^{2}}\right)\right. \\
& \left.+x_{l}^{2}+2 x_{l} \varepsilon+\varepsilon^{2}-2\left(x_{l}+\varepsilon\right)\left(\mu+\frac{\varepsilon}{n}\right)+\mu^{2}+\frac{2 \mu \varepsilon}{n}+\frac{\varepsilon^{2}}{n^{2}}\right] \\
= & \frac{1}{n}\left[\sum_{i=1, i \neq l}^{n}\left(x_{i}^{2}-2 x_{i} \mu-2 x_{i} \frac{\varepsilon}{n}+\mu^{2}+\frac{2 \mu \varepsilon}{n}+\frac{\varepsilon^{2}}{n^{2}}\right)\right. \\
& \left.+x_{l}^{2}+2 x_{l} \varepsilon+\varepsilon^{2}-2 x_{l} \mu-2 x_{l} \frac{\varepsilon}{n}-2 \varepsilon \mu-2 \frac{\varepsilon^{2}}{n}+\mu^{2}+\frac{2 \mu \varepsilon}{n}+\frac{\varepsilon^{2}}{n^{2}}\right] \\
= & \frac{1}{n}\left[\sum_{i=1}^{n}\left(x_{i}^{2}-2 x_{i} \mu-2 x_{i} \frac{\varepsilon}{n}+\mu^{2}+\frac{2 \mu \varepsilon}{n}+\frac{\varepsilon^{2}}{n^{2}}\right)+2 x_{l} \varepsilon+\varepsilon^{2}-2 \varepsilon \mu-2 \frac{\varepsilon^{2}}{n}\right] .
\end{aligned}
$$

We then have that

$$
\begin{aligned}
\triangle I_{V} & (\mathbf{x} ; l, \varepsilon) \\
= & \frac{1}{n}\left[\sum_{i=1}^{n}\left(x_{i}^{2}-2 x_{i} \mu-2 x_{i} \frac{\varepsilon}{n}+\mu^{2}+\frac{2 \mu \varepsilon}{n}+\frac{\varepsilon^{2}}{n^{2}}\right)+2 x_{l} \varepsilon+\varepsilon^{2}-2 \varepsilon \mu-2 \frac{\varepsilon^{2}}{n}\right] \\
& -\frac{1}{n}\left[\sum_{i=1}^{n}\left(x_{i}^{2}-2 x_{i} \mu+\mu^{2}\right)\right] \\
= & \frac{1}{n}\left[\sum_{i=1}^{n}\left(-2 x_{i} \frac{\varepsilon}{n}+\frac{2 \mu \varepsilon}{n}+\frac{\varepsilon^{2}}{n^{2}}\right)+2 x_{l} \varepsilon+\varepsilon^{2}-2 \varepsilon \mu-2 \frac{\varepsilon^{2}}{n}\right] \\
= & \frac{1}{n}\left[-\frac{2 \varepsilon}{n} \sum_{i=1}^{n} x_{i}+2 \mu \varepsilon+\frac{\varepsilon^{2}}{n}+2 x_{l} \varepsilon+\varepsilon^{2}-2 \varepsilon \mu-2 \frac{\varepsilon^{2}}{n}\right] \\
= & \frac{1}{n}\left[-2 \varepsilon \mu+2 \mu \varepsilon+\frac{\varepsilon^{2}}{n}+2 x_{l} \varepsilon+\varepsilon^{2}-2 \varepsilon \mu-2 \frac{\varepsilon^{2}}{n}\right] \\
= & \frac{1}{n}\left(2 x_{l} \varepsilon-2 \varepsilon \mu+\varepsilon^{2}-\frac{\varepsilon^{2}}{n}\right) \\
= & \frac{1}{n}\left(2 \varepsilon\left(x_{l}-\mu\right)+\left(1-\frac{1}{n}\right) \varepsilon^{2}\right) \\
= & \frac{1}{n}\left(2 \varepsilon\left(x_{l}-\mu\right)+\left(\frac{n-1}{n}\right) \varepsilon^{2}\right) .
\end{aligned}
$$

We then have that

$$
\triangle I_{V}(\mathbf{x} ; l, \varepsilon)>0 \Longleftrightarrow 2 \varepsilon\left(x_{l}-\mu\right)>\left(\frac{n-1}{n}\right) \varepsilon^{2}
$$




$$
\begin{aligned}
& \Longleftrightarrow 2 x_{l}-2 \mu>\left(\frac{n-1}{n}\right) \varepsilon \\
& \Longleftrightarrow x_{l}-\mu>\frac{1}{2}\left(\frac{n-1}{n}\right) \varepsilon \\
& \Longleftrightarrow x_{l}>\mu+\frac{1}{2}\left(\frac{n-1}{n}\right) \varepsilon .
\end{aligned}
$$

Thus, $c_{\mathbf{x}, \varepsilon}=\mu+\frac{1}{2}\left(\frac{n-1}{n}\right) \varepsilon$ is a benchmark income and it is clear that $\lim _{\varepsilon \rightarrow 0} c_{\mathbf{x}, \varepsilon}=\mu$. This concludes the proof of part (iv).

Part (v). Take an arbitrary $\mathbf{x} \in \mathbb{R}_{+}^{n}, l \in\{1, \ldots, n\}$, and $\varepsilon>0$. It follows from (5) that

$$
\begin{aligned}
& I_{K}\left(\left(x_{l}+\varepsilon\right)_{l} \mathbf{x}\right)=\frac{1}{n\left(\mu+\frac{\varepsilon}{n}\right)}\left[\sum_{i=1, i \neq l}^{n}\left(x_{i}-\left(\mu+\frac{\varepsilon}{n}\right)\right)^{2}+\left(x_{l}+\varepsilon-\left(\mu+\frac{\varepsilon}{n}\right)\right)^{2}\right] \\
& \triangle I_{K}(\mathbf{x} ; l, \varepsilon)=\frac{1}{n \mu+\varepsilon}\left[\sum_{i=1, i \neq l}^{n}\left(x_{i}-\left(\mu+\frac{\varepsilon}{n}\right)\right)^{2}+\left(x_{l}+\varepsilon-\left(\mu+\frac{\varepsilon}{n}\right)\right)^{2}\right] \\
& -\frac{1}{n \mu} \sum_{i=1}^{n}\left(x_{i}-\mu\right)^{2} \\
& =\frac{1}{n \mu+\varepsilon}\left[\sum_{i=1, i \neq l}^{n}\left(x_{i}^{2}-2 x_{i}\left(\mu+\frac{\varepsilon}{n}\right)+\left(\mu+\frac{\varepsilon}{n}\right)^{2}\right)\right. \\
& \left.+\left(x_{l}+\varepsilon\right)^{2}-2\left(x_{l}+\varepsilon\right)\left(\mu+\frac{\varepsilon}{n}\right)+\left(\mu+\frac{\varepsilon}{n}\right)^{2}\right]-\frac{1}{n \mu} \sum_{i=1}^{n}\left(x_{i}^{2}-2 x_{i} \mu+\mu^{2}\right) \\
& =\frac{1}{n \mu+\varepsilon}\left[\sum_{i=1, i \neq l}^{n}\left(x_{i}^{2}-2 x_{i} \mu-2 x_{i} \frac{\varepsilon}{n}+\mu^{2}+2 \mu \frac{\varepsilon}{n}+\frac{\varepsilon^{2}}{n^{2}}\right)\right. \\
& \left.+x_{l}^{2}+2 x_{l} \varepsilon+\varepsilon^{2}-2 x_{l} \mu-2 x_{l} \frac{\varepsilon}{n}-2 \varepsilon \mu-2 \frac{\varepsilon^{2}}{n}+\mu^{2}+2 \mu \frac{\varepsilon}{n}+\frac{\varepsilon^{2}}{n^{2}}\right] \\
& -\frac{1}{n \mu} \sum_{i=1}^{n}\left(x_{i}^{2}-2 x_{i} \mu+\mu^{2}\right) \\
& =\frac{1}{n \mu+\varepsilon}\left[\sum_{i=1}^{n}\left(\mu^{2}+2 \mu \frac{\varepsilon}{n}+\frac{\varepsilon^{2}}{n^{2}}\right)+\sum_{i=1, i \neq l}^{n}\left(x_{i}^{2}-2 x_{i} \mu-2 x_{i} \frac{\varepsilon}{n}\right)\right. \\
& \left.+x_{l}^{2}-2 x_{l} \mu-2 x_{l} \frac{\varepsilon}{n}+2 x_{l} \varepsilon+\varepsilon^{2}-2 \varepsilon \mu-2 \frac{\varepsilon^{2}}{n}\right]-\frac{1}{n \mu} \sum_{i=1}^{n}\left(x_{i}^{2}-2 x_{i} \mu+\mu^{2}\right) \\
& =\frac{1}{n \mu+\varepsilon}\left[n\left(\mu^{2}+2 \mu \frac{\varepsilon}{n}+\frac{\varepsilon^{2}}{n^{2}}\right)+\sum_{i=1}^{n}\left(x_{i}^{2}-2 x_{i} \mu-2 x_{i} \frac{\varepsilon}{n}\right)+2 x_{l} \varepsilon+\varepsilon^{2}-2 \varepsilon \mu-2 \frac{\varepsilon^{2}}{n}\right] \\
& -\frac{1}{n \mu} \sum_{i=1}^{n}\left(x_{i}^{2}-2 x_{i} \mu+\mu^{2}\right)
\end{aligned}
$$




$$
\begin{aligned}
& =\frac{1}{n \mu+\varepsilon}\left[n \mu^{2}+2 \mu \varepsilon+\frac{\varepsilon^{2}}{n}+\sum_{i=1}^{n}\left(x_{i}^{2}-2 x_{i} \mu-2 x_{i} \frac{\varepsilon}{n}\right)+2 x_{l} \varepsilon+\varepsilon^{2}-2 \mu \varepsilon-2 \frac{\varepsilon^{2}}{n}\right] \\
& -\frac{1}{n \mu} \sum_{i=1}^{n}\left(x_{i}^{2}-2 x_{i} \mu+\mu^{2}\right) \\
& =\frac{1}{n \mu+\varepsilon}\left[n \mu^{2}+\varepsilon^{2}\left(1-\frac{1}{n}\right)+2 x_{l} \varepsilon+\sum_{i=1}^{n} x_{i}^{2}-2 \mu \sum_{i=1}^{n} x_{i}-2 \varepsilon \sum_{i=1}^{n} \frac{x_{i}}{n}\right] \\
& -\frac{1}{n \mu}\left[\sum_{i=1}^{n} x_{i}^{2}-2 \mu \sum_{i=1}^{n} x_{i}+n \mu^{2}\right] \\
& =\frac{1}{n \mu+\varepsilon}\left[n \mu^{2}+\varepsilon^{2}\left(1-\frac{1}{n}\right)+2 x_{l} \varepsilon+\sum_{i=1}^{n} x_{i}^{2}-2 n \mu^{2}-2 \varepsilon \sum_{i=1}^{n} \frac{x_{i}}{n}\right] \\
& -\frac{1}{n \mu}\left[\sum_{i=1}^{n} x_{i}^{2}-2 n \mu^{2}+n \mu^{2}\right] \\
& =\frac{1}{n \mu+\varepsilon}\left[-n \mu^{2}+\varepsilon^{2}\left(1-\frac{1}{n}\right)+2 x_{l} \varepsilon+\sum_{i=1}^{n} x_{i}^{2}-2 \varepsilon \sum_{i=1}^{n} \frac{x_{i}}{n}\right]-\frac{1}{n \mu}\left[\sum_{i=1}^{n} x_{i}^{2}-n \mu^{2}\right] \\
& =\left(\sum_{i=1}^{n} x_{i}^{2}-n \mu^{2}\right)\left(\frac{1}{n \mu+\varepsilon}-\frac{1}{n \mu}\right)+\frac{1}{n \mu+\varepsilon}\left[\varepsilon^{2}\left(1-\frac{1}{n}\right)+2 x_{l} \varepsilon-2 \varepsilon \sum_{i=1}^{n} \frac{x_{i}}{n}\right] \\
& =\left(\sum_{i=1}^{n} x_{i}^{2}-n \mu^{2}\right)\left(\frac{n \mu-(n \mu+\varepsilon)}{n \mu(n \mu+\varepsilon)}\right)+\frac{1}{n \mu+\varepsilon}\left[\varepsilon^{2}\left(1-\frac{1}{n}\right)+2 x_{l} \varepsilon-2 \frac{\varepsilon}{n} \cdot n \mu\right] \\
& =\left(\sum_{i=1}^{n} x_{i}^{2}-n \mu^{2}\right)\left(\frac{-\varepsilon}{n \mu(n \mu+\varepsilon)}\right)+\frac{1}{n \mu+\varepsilon}\left[\varepsilon^{2}\left(1-\frac{1}{n}\right)+2 x_{l} \varepsilon-2 \varepsilon \mu\right] \\
& =n \sigma_{\mathbf{x}}^{2}\left(\frac{-\varepsilon}{n \mu(n \mu+\varepsilon)}\right)+\frac{\varepsilon}{n \mu+\varepsilon}\left[\frac{\varepsilon(n-1)}{n}+2 x_{l}-2 \mu\right] \\
& =\frac{\varepsilon}{n \mu+\varepsilon}\left(2\left(x_{l}-\mu\right)-\frac{\sigma_{\mathbf{x}}^{2}}{\mu}+\frac{\varepsilon(n-1)}{n}\right) \text {. }
\end{aligned}
$$

We then have that

$$
\begin{aligned}
\Delta I_{K}(\mathbf{x} ; l, \varepsilon)>0 & \Longleftrightarrow-\frac{\sigma_{\mathbf{x}}^{2}}{\mu}+\frac{\varepsilon(n-1)}{n}+2 x_{l}-2 \mu>0 \\
& \Longleftrightarrow 2 x_{l}>2 \mu+\frac{\sigma_{\mathbf{x}}^{2}}{\mu}-\frac{\varepsilon(n-1)}{n} \\
& \Longleftrightarrow x_{l}>\mu+\frac{\sigma_{\mathbf{x}}^{2}}{2 \mu}-\frac{\varepsilon(n-1)}{2 n} .
\end{aligned}
$$


Thus, $c_{\mathbf{x}, \varepsilon}=\mu+\frac{\sigma_{\mathbf{x}}^{2}}{2 \mu}-\frac{\varepsilon(n-1)}{2 n}$ is a benchmark income and it is clear that $\lim _{\varepsilon \rightarrow 0} c_{\mathbf{x}, \varepsilon}=$ $\mu+\frac{\sigma_{\mathbf{x}}^{2}}{2 \mu}$. This concludes the proof of part (v) and so too of Proposition 1 .

\section{B Proof of Theorem 1}

Let $\mathbf{x} \in \mathbb{R}_{+}^{n}$ and $\delta>0$ be arbitrary. First, suppose that for some $k \in\{1, \ldots, n\}$ we have $\left(x_{k}+\delta\right)_{k} \mathbf{x} \preccurlyeq \mathbf{x}$. Consider any $x_{k^{\prime}}>x_{k}$ (and so $k^{\prime}>k$ ) satisfying the requirement that either $k^{\prime}=n$ or $x_{k^{\prime}} \leq x_{k^{\prime}+1}-\delta$.

Assumption 1 Suppose that $\left(x_{k^{\prime}}+\delta\right)_{k^{\prime}} \mathbf{x} \succcurlyeq \mathbf{x}$.

Strong transfer implies that $\left(x_{k}+\delta^{\prime}\right)_{k}\left(x_{k^{\prime}}+\delta-\delta^{\prime}\right)_{k^{\prime}} \mathbf{x} \succ\left(x_{k^{\prime}}+\delta\right)_{k^{\prime}} \mathbf{x}$ for all $\delta^{\prime} \in(0, \delta]$. In particular, for $\delta^{\prime}=\delta$, we have $\left(x_{k}+\delta\right)_{k} \mathbf{x} \succ\left(x_{k^{\prime}}+\delta\right)_{k^{\prime}} \mathbf{x}$. By transitivity, it follows that $\left(x_{k}+\delta\right)_{k} \mathbf{x} \succ \mathbf{x}$. But this contradicts the initial supposition that $\left(x_{k}+\delta\right)_{k} \mathbf{x} \preccurlyeq \mathbf{x}$. Thus Assumption 1 cannot be true. We conclude that for all $x_{k^{\prime}}>x_{k}$ we have $\left(x_{k^{\prime}}+\delta\right)_{k^{\prime}} \mathbf{x} \prec \mathbf{x}$.

We know that $\left(x_{k}+\delta\right)_{k} \mathbf{x} \preccurlyeq \mathbf{x}$. Suppose that indifference holds and $\left(x_{k}+\delta\right)_{k} \mathbf{x} \sim \mathbf{x}$. Consider any arbitrary $x_{j}<x_{k}$ (and thus $j<k$ ) satisfying the requirement that $x_{j} \leq x_{j+1}-\delta$.

Then, strong transfer implies that $\left(x_{j}+\delta^{\prime}\right)_{j}\left(x_{k}+\delta-\delta^{\prime}\right)_{k} \mathbf{x} \succ\left(x_{k}+\delta\right)_{k} \mathbf{x}$ for all $\delta^{\prime} \in(0, \delta]$. In particular, for $\delta^{\prime}=\delta$, we have $\left(x_{j}+\delta\right)_{j} \mathbf{x} \succ\left(x_{k}+\delta\right)_{k} \mathbf{x}$. By transitivity, it follows that $\left(x_{j}+\delta\right)_{j} \mathbf{x} \succ \mathbf{x}$ for all $x_{j}<x_{k}$ satisfying the requirement that $x_{j} \leq x_{j+1}-\delta$.

Then setting $c_{\mathbf{x}, \delta}=x_{k}$, statements (1) and (2) of Theorem 1 are both satisfied.

Suppose instead that $\left(x_{k}+\delta\right)_{k} \mathbf{x} \prec \mathbf{x}$. We then have that $\left(x_{k^{\prime}}+\delta\right)_{k^{\prime}} \mathbf{x} \prec \mathbf{x}$ for all $x_{k^{\prime}} \geq x_{k}$. Let $i^{*} \in\{1, \ldots, n\}$ be the smallest index such that $x_{i^{*}} \leq x_{i^{*}+1}-\delta$ and $\left(x_{i^{*}}+\delta\right)_{i^{*}} \mathbf{x} \preceq \mathbf{x}$. Then set $c_{\mathbf{x}, \delta}=x_{i^{*}}$. If $i^{*}=1$ then statement (1) in Theorem 1 holds trivially. Statement (2) in Theorem 1 is seen to hold by applying similar steps as above (i.e., by assuming that there exists $i>i^{*}$ such that $\left(x_{i}+\delta\right)_{i} \mathbf{x} \succcurlyeq \mathbf{x}$, finding a contradiction and concluding that for all $i>i^{*}$ (and so all $x_{i}>c_{\mathbf{x}, \delta}$ ) such that $x_{i} \leq x_{i+1}-\delta$ or $i=n$ we have $\left(x_{i}+\delta\right)_{i} \mathbf{x} \prec \mathbf{x}$.) If $i^{*}>1$ then $\left(x_{i}+\delta\right)_{i} \mathbf{x} \succ \mathbf{x}$ for all $i<i^{*}$ (and so for all $x_{i}<c_{\mathbf{x}, \delta}$ ) such that $x_{i} \leq x_{i+1}-\delta$-i.e., statement (1) in Theorem 1 holds. Statement (2) in Theorem 1 holds by the same reasoning as in the case for $i^{*}=1$.

Finally, suppose that there exists no $k \in\{1, \ldots, n\}$ where $\left(x_{k}+\delta\right)_{k} \mathbf{x} \preccurlyeq \mathbf{x}$. Then, for all $k \in\{1, \ldots, n\}$ satisfying the requirement that $k=n$ or $x_{k} \leq x_{k+1}-\delta$, $\left(x_{k}+\delta\right)_{k} \mathbf{x} \succ \mathbf{x}$. By setting $c_{\mathbf{x}, \delta}=\infty$ (or any value larger than $x_{n}+\delta$ ) statement (1) of the theorem is satisfied; and trivially also statement (2).

This concludes the proof of Theorem 1 .

\section{Proof of Theorem 2}

Let $\mathbf{x} \in \mathbb{R}_{+}^{n}$ be arbitrary. From Theorem 1 it follows that for any arbitrary $\delta_{1}, \delta_{2}>0$, there exist $c_{\mathbf{X}, \delta_{\mathbf{1}}}$ and $c_{\mathbf{x}, \delta_{\mathbf{2}}}$ such that each of the following hold: 
(a) If $x_{i}<c_{\mathbf{x}, \delta_{\mathbf{1}}}$ then $\left(x_{i}+\delta_{1}\right)_{i} \mathbf{x} \succ \mathbf{x}$.

(b) If $x_{i}<c_{\mathbf{x}, \delta_{\mathbf{2}}}$ then $\left(x_{i}+\delta_{2}\right)_{i} \mathbf{x} \succ \mathbf{x}$.

(c) If $x_{i}>c_{\mathbf{x}, \delta_{1}}$ we have $\left(x_{i}+\delta_{1}\right)_{i} \mathbf{x} \prec \mathbf{x}$.

(d) If $x_{i}>c_{\mathbf{x}, \delta_{2}}$ then $\left(x_{i}+\delta_{2}\right)_{i} \mathbf{x} \prec \mathbf{x}$.

Applying Positional consistency to each of (a), (b), (c), and (d), we have that each of the following hold:

(a') If $x_{i}<c_{\mathbf{x}, \delta_{\mathbf{1}}}$ then $\left(x_{i}+\delta\right)_{i} \mathbf{x} \succ \mathbf{x}$ for all $\delta>0$.

(b') If $x_{i}<c_{\mathbf{x}, \delta_{\mathbf{2}}}$ then $\left(x_{i}+\delta\right)_{i} \mathbf{x} \succ \mathbf{x}$ for all $\delta>0$.

(c') If $x_{i}>c_{\mathbf{x}, \delta_{\mathbf{1}}}$ we have $\left(x_{i}+\delta\right)_{i} \mathbf{x} \prec \mathbf{x}$ for all $\delta>0$.

(d') If $x_{i}>c_{\mathbf{x}, \delta_{\mathbf{2}}}$ then $\left(x_{i}+\delta\right)_{i} \mathbf{x} \prec \mathbf{x}$ for all $\delta>0$.

First suppose that $c_{\mathbf{x}, \delta_{\mathbf{1}}} \neq c_{\mathbf{x}, \delta_{\mathbf{2}}}$. Without loss of generality, we may assume that $c_{\mathbf{x}, \delta_{1}}<c_{\mathbf{x}, \delta_{2}}$. Since each of (a'), (b'), (c'), and (d') hold, there can not exist any $x_{i} \in \mathbf{x}$ such that $c_{\mathbf{x}, \delta_{1}}<x_{i}<c_{\mathbf{x}, \delta_{2}}$, because this would imply that, for all $\delta>0$, both $\left(x_{i}+\delta\right)_{i} \mathbf{x} \succ \mathbf{x}$ and $\left(x_{i}+\delta\right)_{i} \mathbf{x} \prec \mathbf{x}$ hold, which is not possible. Now choose any $c_{\mathbf{x}} \in\left(c_{\mathbf{x}, \delta_{1}}, c_{\mathbf{x}, \delta_{2}}\right)$. Since $c_{\mathbf{x}}<c_{\mathbf{x}, \delta_{\mathbf{2}}}$, it follows from (b') that:

(i) for all $x_{i}<c_{\mathbf{x}}$ we have $\left(x_{i}+\delta\right)_{i} \mathbf{x} \succ \mathbf{x}$ for all $\delta>0$.

Since $c_{\mathbf{x}}>c_{\mathbf{x}, \delta_{1}}$, it follows from (c') that

(ii) for all $x_{i}>c_{\mathbf{x}}$ we have $\left(x_{i}+\delta\right)_{i} \mathbf{x} \prec \mathbf{x}$ for all $\delta>0$.

Thus, statements (1) and (2) of Theorem 2 are both satisfied.

Now suppose instead that $c_{\mathbf{x}, \delta_{\mathbf{1}}}=c_{\mathbf{x}, \delta_{\mathbf{2}}}$. Set $c_{\mathbf{x}}=c_{\mathbf{x}, \delta_{\mathbf{1}}}=c_{\mathbf{x}, \delta_{\mathbf{2}}}$. Statements (1) and

(2) of Theorem 2 then follow immediately from (a') and (c') respectively, i.e.,

(i) for all $x_{i}<c_{\mathbf{x}}$ we have $\left(x_{i}+\delta\right)_{i} \mathbf{x} \succ \mathbf{x}$ for all $\delta>0$.

(ii) for all $x_{i}>c_{\mathbf{x}}$ we have $\left(x_{i}+\delta\right)_{i} \mathbf{x} \prec \mathbf{x}$ for all $\delta>0$.

This concludes the proof of Theorem 2 .

\section{References}

Atkinson, A.: Social justice and public policy. MIT Press, Cambridge (1983)

Corvalan, A.: The impact of a marginal subsidy on Gini indices. Rev Income Wealth 60, 596-603 (2014)

Hoffmann, R.: Effect of the rise of a person's income on inequality. Brazilian Rev Econom 21, 237-262 (2001)

Lambert, P., Lanza, G.: The effect on inequality of changing one or two incomes. J Econom Inequal 4, 253-277 (2006)

Sen, A.: Poverty: an ordinal approach to measurement. Econometrica 44, 219-231 (1976) 